(C) 2016 IEEE. Personal use of this material is permitted. Permission from IEEE must be obtained for all other uses, in any current or future media, including reprinting/republishing this material for advertising or promotional purposes, creating new collective works, for resale or redistribution to servers or lists, or reuse of any copyrighted component of this work in other works. 


\title{
Microwave Doppler Tomography of High Impedance Ground Planes for Aerospace Applications
}

\author{
Kelvin J. Nicholson \\ Aerospace Division \\ Defence Science and Technology Group \\ Melbourne, Australia \\ kelvin.nicholson@dsto.defence.gov.au
}

\author{
Thomas Baum and Kamran Ghorbani \\ School of Electrical and Computer Engineering \\ RMIT University \\ Melbourne, Australia \\ thomas.baum@rmit.edu.au
}

\author{
Richard W. Ziolkowski \\ Department of Electrical and Computer Engineering \\ University of Arizona \\ Tucson, AZ, USA \\ ziolkowski@ece.arizona.edu
}

\begin{abstract}
Recent developments in the areas of metamaterials and high impedance ground planes (HIGPs) have created new opportunities for the development of novel multi-functional aerospace materials. One niche application of such materials is the suppression of electromagnetic surface waves across the outer mold line (OML) of aerospace structures. Doppler tomographic imaging and spectral filtering techniques are used to characterize the performance of a simple numerically simulated HIGP. A tapered HIGP concept is introduced to improve the performance over a range of illumination angles. This tapered HIGP is to be embroidered in a commercial aerospace pre-preg material. Experimental results will be presented at the conference.
\end{abstract}

Keywords-Aerospace materials, composite materials, high impednace groudn plane, metamaterials, tomography.

\section{INTRODUCTION}

Mechanical repairs to the outer mold line (OML) surface of modern aerospace structures are designed to ensure the electromagnetic properties of adjacent RF systems are not perturbed. For example, consider a simple aircraft antenna (mounted on the OML) that excites a surface wave (defined by $J$ ) in Fig. 1. This surface wave may scatter from the OML repair thereby potentially degrading the electromagnetic performance of the antenna. Similarly, adjacent antenna systems may inadvertently couple as a result of the excitation of a surface wave across the OML.

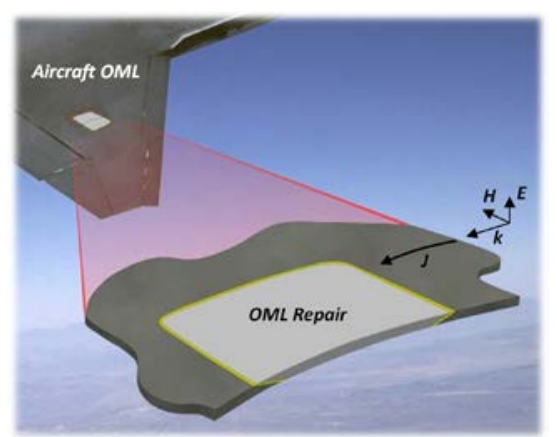

Fig. 1. Representative OML repair illustrating the interaction with a surface wave. The electrical impedance miss-match between the aircraft OML and repair material will result in a scattered wave.
Recent developments in the areas of metamaterials and high impedance ground planes (HIGPs) have created new opportunities for the development of novel multi-functional aerospace materials [1]. This paper investigates how these emerging technologies can be integrated into modern aerospace composite structures for the purpose of controlling the propagation of electromagnetic surface waves. A microwave Doppler tomographic technique will be used to characterize the performance of novel multi-functional structures incorporating HIGPs for the purpose of surface wave suppression.

\section{Metamaterial Design}

Metamaterials are constructed by embedding subwavelength inclusions into a host medium to form a one (metaline), two (meta-surface) or three (bulk metamaterial) dimensional volume. These inclusions act analogous to artificial molecules that can be engineered to produce the desired bulk material response. In this regard, the metamaterial gains its properties not only from its structure but also from its composition. Consequently it is possible to engineer the dispersion within a metamaterial to inhibit the propagation of surface waves. One example of a two-dimensional metamaterial capable of suppressing the propagation of a surface wave is the high-impedance ground plane (HIGP) [2].

To illustrate how the HIGP concept may aid in the suppression of surface waves, consider the simple rectangular mushroom HIGP unit cell model illustrated in Fig. 2.

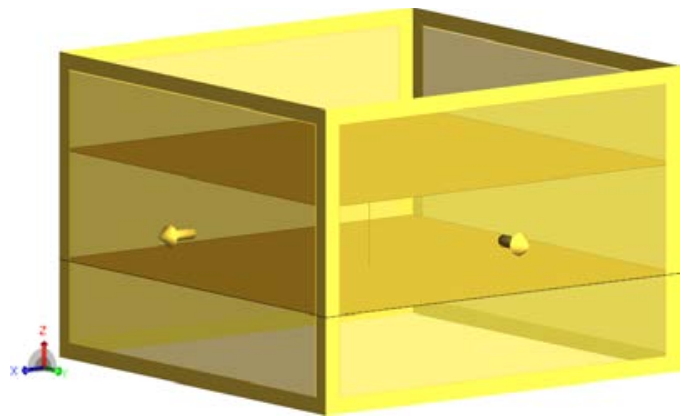

Fig. 2. Proposed unit cell of the HIGP. Periodic boundary conditions are present on the $x-z$ and $y$ - $z$ boundaries. 
This unit cell measures $10.0 \times 10.0 \mathrm{~mm}^{2}$ with a $0.2 \mathrm{~mm}$ gap between its PEC patches. The cylindrical PEC post measures $0.05 \mathrm{~mm}$ in diameter with a total height of $2.5 \mathrm{~mm}$. No dielectric material is present to simplify the numerical simulation. The HIGP exhibits a reflected phase between \pm $90.0^{\circ}$ over the bandwidth $5.75 \mathrm{GHz}$ to $8.25 \mathrm{GHz}$ as illustrated by the simulation results given in Fig. 3 . It is anticipated that this simple HIGP will suppress the propagation of a surface wave over most of this $5.75 \mathrm{GHz}$ to $8.25 \mathrm{GHz}$ bandwidth.

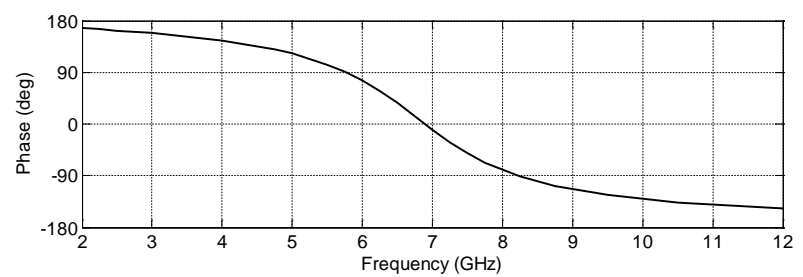

Fig. 3. Reflected phase for the simple HIGP in Fig. 2.

\section{DOPPLER TOMOGRAPHY}

The reconstruction of a target from a set of its projections is a classical problem that is open to a variety of solution methods as reviewed in [3]. For this OML surface application, a simple Doppler tomographic process was selected. Consider the geometry illustrated in Fig. 3.

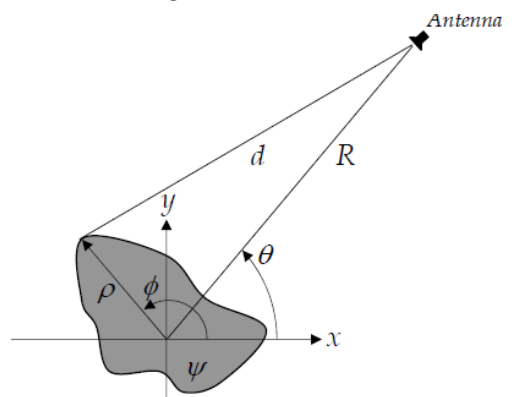

Fig. 4. Tomographic reconstruction geometry [4].

The tomographic image for a single illumination frequency is given by the equation;

$$
\hat{\psi}(\rho, \phi \mid f)=\frac{4 f_{0}}{c^{2}} \mathcal{F}^{-1}\left\{\mathcal{F}\{S(\theta \mid f)\} \times \mathcal{F}\left\{e^{-j \frac{4 \pi f_{0}}{c} \rho \cos (\phi)}\right\}\right\}
$$

where $\mathrm{S}(\theta)$ is the measured scattering data at frequency $f$ and $\hat{\psi}$ is the approximation of the target reflectivity function $(\psi)$. A composite image may then be generated by summation over $N_{f}$ frequencies according to the equation:

$$
\hat{\psi}(\rho, \phi)=\Delta f \sum_{n=1}^{N_{f}} \hat{\psi}\left(\rho, \phi \mid f_{n}\right)
$$

This composite image achieves a significant improvement in dynamic range. The ability to resolve an image utilizing either a single frequency (Equation 1) or a bandwidth (Equation 2) is invaluable for assessing typically narrow-band structures such as the HIGP illustrated in Fig 2. For example, consider the 400 x $400 \mathrm{~mm}^{2}$ PEC parent surface with a 100 x $100 \times 2.5 \mathrm{~mm}^{3}$ PEC patch that is illustrated in Fig. 5.

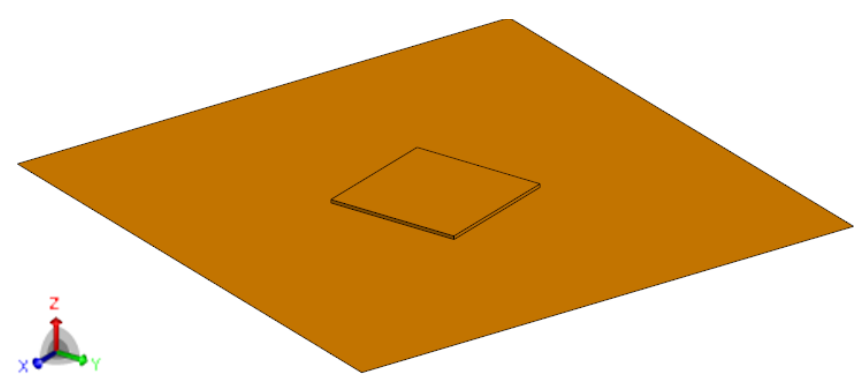

Fig. 5. Simulated target with representative patch repair.

The target in Fig. 5 was illuminated numerically by a plane wave (polarized along the $z$ axis) from an altitude of $10^{\circ}$ (from the $x-y$ plane). A tomographic image was reconstructed over the HIGP bandwidth expected to suppress the propagation of surface waves. The resulting image is illustrated in Fig. 6.

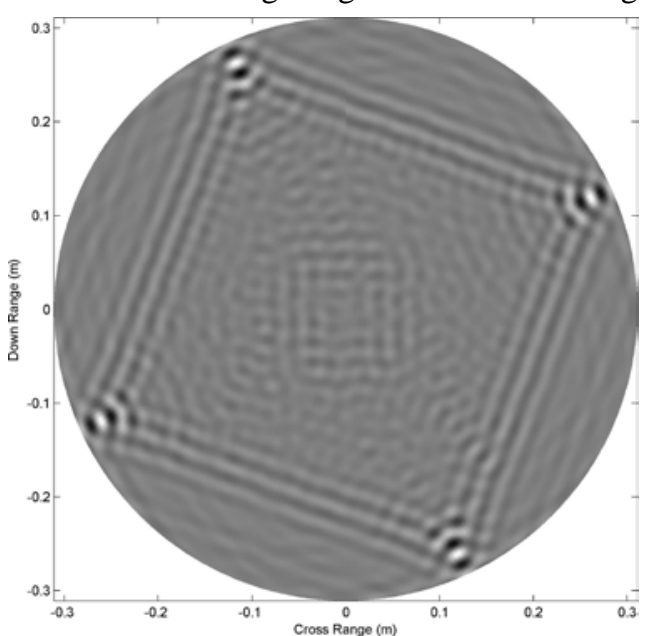

Fig. 6. Normalised tomographic reconstruction of the geometry in Fig. 5.

The parent edges are evident with the strongest scattering occurring at the corners. This outcome is a direct consequence of the multi-path bounce of the surface waves from the dihedral formed by the parent edges. Furthermore, scattering from the patch is also clearly evident, albeit at a much lower magnitude. The HIGP introduced in Section II was then applied to the region surrounding the patch as illustrated in Fig. 7.



Fig. 7. Simulated target with representative patch repair and HIGP. 
Note the deliberate orientation of the patch $\left(0^{\circ}\right)$, HIGP region $\left(50^{\circ}\right)$ and parent $\left(70^{\circ}\right)$ relative to the $x$-axis in Fig. 7 . Comparison of the radar cross section (RCS) of the geometries in Fig. 5 (denoted 'No HIGP') and Fig. 7 (denoted 'With HIGP') illustrates the difficulty in determining the contributions of the patch and the HIGP. Clearly, the scattering of the parent edges (i.e. the peak at $70^{\circ}$ ) dominates the total RCS.



Fig. 8. Comparrison of the normalised RCS at the centre frequency for the geometry in Fig. 5 and Fig. 7.

However, a spectral filtering technique [5] may be applied to the scattering data to isolate the patch and HIGP regions from the parent RCS while retaining the contribution of the excited surface wave. This filtering technique is applied to a circular region surrounding the patch. This region is marked on the tomographic image in Fig. 9.



Fig. 9. Normalised tomographic reconstruction of the geometry in Fig. 7 with the filtered region identified (red circle).

The scattering associated with the patch and HIGP regions is clearly evident in Fig. 9. Applying the filter to the scattering data, the RCS of the patch and HIGP (denoted 'With HIGP') is revealed. The results are given in Fig. 10. The same filtering process can also be applied to the scattering data in Fig. 6 to yield the RCS of just the patch. The result (denoted 'No HIGP') is also reported in Fig. 10.



Fig. 10. Comparrison of the normalised RCS of each gated geometry at the centre frequency.
The RCS of the patch at $0^{\circ}$ has been significantly reduced by the addition of the HIGP region confirming the suppression of the surface wave for this illumination direction. However, at $50^{\circ}$ the wave impedance miss-match between the parent and HIGP regions is responsible for the increased RCS. This suggests that a tapered HIGP design, which would provide a broader angular interval of suppression, may further reduce the scattering associated with the excited surface wave. One such tapered HIGP concept is illustrated in Fig. 11.

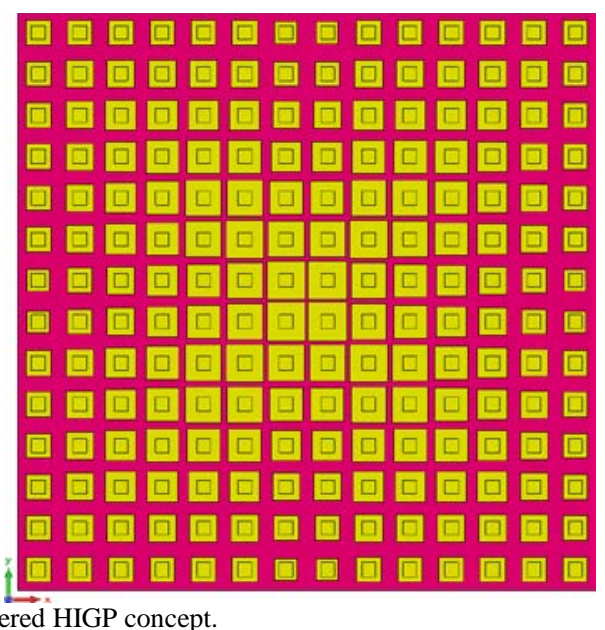

Fig. 11. Tapered HIGP concept

This tapered HIGP concept is anticipated to suppress the surface wave for most, if not all, illumination angles. When applied to an OML repair, the expectation is for a significant scattering reduction. However, given the size of the structure and the need for a dielectric medium, numerical simulation for tomographic reconstruction is computationally prohibitive. Hence, experimental validation was required to prove the concept.

\section{MANUFACTURE}

The embroidery of conductive threads into traditional textiles is well established for the purpose of integrating circuits, sensors and antennas [6]. However, the increasing use of carbon and glass reinforced composites in modern aircraft has resulted in a new class of materials referred to as 'prepregs'. These pre-preg materials consist of a fabric (i.e. glass, carbon or a hybrid of both) pre-impregnated with the optimum amount of resin matrix. This resin matrix has been partially cured (or B-staged) such that the pre-preg remains flexible for laminating over a mold. Once all the pre-preg plies have been stacked appropriately, the composite part is typically cured in an autoclave at temperatures in excess of $170^{\circ} \mathrm{C}$ and pressures exceeding $700 \mathrm{kPa}$ to yield a aerospace-grade structural component.

These pre-preg materials lend themselves to the embroidery process thereby allowing novel electromagnetic features to be sewn directly into the composite laminate prior to autoclave curing. The resulting multifunctional composite laminates may find applications in a variety of systems that are currently found on many conformal aerospace structures, such as the wings and fuselage. For example, antennas, feed networks and sensors may be integrated into the OML by combining this 
embroidery process with existing aerospace manufacturing techniques. This work is concerned with the integration of the proposed tapered HIGP structure presented in Section II for the purpose of suppressing the propagation of surface waves across the OML. This has the advantage of minimizing cross-talk or the impact of conventional mechanical repairs on the electromagnetic performance of adjacent antenna systems.

Commercial Hexply glass (48 g.m ${ }^{-2}$ ) impregnated with Hexcel 914E epoxy resin was selected for the pre-preg material. A silver plated Kevlar fiber manufactured by Aracon (Part No. XS0200E-025) was selected as the conductive thread. The embroidery of the HIGP was performed on a Brother PR1000 numerically controlled embroidery machine. The tapered HIGP being sewn into this aerospace pre-preg is illustrated in Fig. 7.

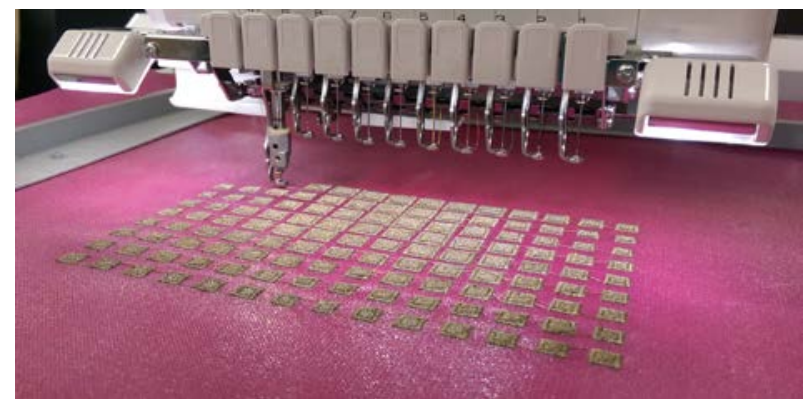

Fig. 12. Embroidery of tapered HIGP surface into aerospace pre-preg material.

The embroidered pre-preg ply containing the patch geometry (illustrated in Fig. 11) was de-bulked against a 30 ply glass fiber reinforced laminate to form the dielectric substrate. Rectangular vias were then embroidered through the whole laminate. The resulting HIGP was autoclave cured against a carbon fiber reinforced quasi-isotropic panel. The experimental characterization of this tapered HIGP structure will be presented at the conference.

\section{CONCLUSION}

This paper has numerically assessed a simple HIGP layer for the purpose of suppressing the propagation of surface waves across the OML surface of an aircraft. This has the advantage of minimizing cross-talk or the impact of conventional mechanical repairs on the electromagnetic performance of adjacent antenna systems. A Doppler tomographic process together with a spectral filtering technique was applied to isolate the electromagnetic scattering associated with the patch and the HIGP from the parent structure. The numerical simulations confirmed the suppression of the surface wave and the corresponding RCS reduction over a limited range of illumination angles. This highlighted the need for a tapered HIGP geometry to increase this angular region. The proposed tapered HIGP was successfully embroidered into a commercial aerospace pre-preg material and applied to a representative aircraft OML surface. The experimental characterization of this multifunctional structure will be presented at the conference.

\section{REFERENCES}

[1] K. J. Nicholson, J. D’Cruz, and P. J. Callus, "Multifunctional Structures for Australian Defence Force land, Sea and Air Platforms,” DSTO-TR-2390, 2010.

[2] D. Sievenpiper, L. Zhang, R. F. J. Broas, N. G. Alexopolous, and E. Yablonovitch, "High-impedance electromagnetic surfaces with a forbidden frequency band," IEEE Trans. Microw. Theory Tech., vol. 47, no. 11, pp. 2059-2074, Nov. 1999.

[3] R. M. Mersereau and A. V. Oppenheim, "Digital reconstruction of multidimensional signals from their projections,” Proc. IEEE, vol. 62, no. 10, pp. 1319-1338, Oct. 1974.

[4] K. J. Nicholson and C. H. Wang, "Improved Near-Field Radar Cross-Section Measurement Technique," IEEE Antennas Wirel. Propag. Lett., vol. 8, pp. 1103-1106, 2009.

[5] A. V. Oppenheim, R. W. Schafer, and J. R. Buck, Discrete-Time Signal Processing, 2 Edition. Upper Saddle River, N.J.: Prentice Hall, 1999.

[6] H. Khalee, Innovation in Wearable and Flexible Antennas. Southampton, UK: WIT Press, 2014. 